\title{
Tendencias teóricas emergentes del desarrollo organizacional
}

\author{
Fabara, Xavier* \\ https://orcid.org/0000-0001-8299-1036 \\ *Universidad de las Fuerzas Armadas ESPE, Sangolquí, Ecuador \\ E-mail:xhfabara@espe.edu.ec \\ Recibido: 5 de enero de 2021 \\ Aprobado: 20 de enero de 2021
}

\section{Resumen}

Los sistemas organizaciones han evolucionado a la par de los procesos sociales, organizaciones que son el cimiento de toda sociedad, agrupaciones que deliberadamente realizan las personas para el logro de algún propósito en específico. En tal sentido es importante revisar y evaluar la perspectiva teórica sobre las organizaciones fruto de las interrelaciones sociales, sus enfoques para comprender el comportamiento de sus estructuras, el ámbito de acción su pensamiento así como sus restricciones; por tal motivo el objetivo del presente trabajo es realizar una investigación descriptiva - documental en base a la revisión de importantes aportes sobre los modelos de los Estudios Organizacionales que se abordaran desde la perspectiva de los ambientes donde se desenvuelven como el modelo de ecología de poblaciones, el modelo de dependencia de recursos, así como también modelos de acción organizacional, con énfasis en la dirección hacia objetivos como el modelo de la contingencia racional y el modelo de costos de las transacciones, y por último la perspectiva sobre los actores organizacionales a través de sus valores canalizando el modelo institucional de la organizaciones, a través de una metodología de abordaje que se fundamenta en la Desconstrucción y Reconstrucción teórica por Van Djik; para establecer una mejor cosmovisión de la investigación a ser estudiada y determinar en sus conclusiones las proyecciones y tendencias del desarrollo organizacional basadas en un sistema cambiante más abiertos y flexibles acordes a los avances tecnológicos y competitivos del mercado.

Palabras clave: Organización, Tendencias, Modelos Organizacionales, Administración 


\title{
Emerging theoretical trends of organizational development
}

\begin{abstract}
Organizational systems have evolved along with social processes, organizations that are the foundation of every society, groups that people deliberately create to achieve a specific purpose. In this sense, it is important to review and evaluate the theoretical perspective on organizations as a result of social interrelations, their approaches to understanding the behavior of their structures, the scope of action, their thinking, as well as their restrictions; For this reason, the objective of this work is to carry out a descriptive-documentary research based on the review of important contributions on the models of Organizational Studies that will be approached from the perspective of the environments where they develop as the model of population ecology, the resource dependency model, as well as organizational action models, with emphasis on the direction towards objectives such as the rational contingency model and the transaction costs model, and finally the perspective on the organizational actors through its values channeling the institutional model of the organizations, through an approach methodology that is based on the theoretical Deconstruction and Reconstruction by Van Djik; to establish a better worldview of the research to be studied and determine in its conclusions the projections and trends of organizational development based on a more open and flexible changing system according to the technological and competitive advances of the market.
\end{abstract}

Keywords: Organization, Trends, Organizational Models, Administration

\section{Introducción}

Existen muchas definiciones de tendencias, toda tendencia es un conjunto de señales de cambio que apunta hacia cierta dirección. Es un patrón de significado, creado por indicios y pistas de cierto cambio. Las tendencias, aunque novedosas, tienen ya cierto peso de evidencia histórico. En este caso se encuentran tendencias de estudios organizacionales.

los Estudios Organizacionales se cita: "serie de conversaciones, en particular aquellas de los investigadores del fenómeno organizacional, quienes ayudan a definir las organizaciones a través de términos derivados de paradigmas, métodos $\mathrm{y}$ suposiciones, ellos mismos derivados de anteriores conversaciones" (Clegg y Hardy, 1996, p.3). Los estudios organizacionales tienen su ámbito de análisis a las organizaciones, el análisis de diversos fenómenos sociales, culturales, políticos, económicos, etc.

\section{Metodología}

El trabajo se realizó en base a una revisión bibliográfica y documental sobre las fuentes de datos digitales, utilizando el análisis de artículos científicos los cuales se procedieron a seleccionar de acuerdo a la utilidad para la investigación, se ordenaron por temática y se realizó un análisis de contenidos; buscando las ideas expresadas en cada uno de los documentos.

Sin embargo, es importante tener en cuenta que los documentos son muy variados, teniendo que recurrir a todos aquellos que demande nuestro problema, sin más limitaciones que su pertinencia y su posibilidad de acceso (Marín Ibáñez, 1985).

Además, se incorporó la metodología de abordaje que se fundamenta en la Desconstrucción y Reconstrucción teórica por Van Djik. Para poder establecer una mejor cosmovisión de la investigación a ser estudiada, la misma esta contempla dos fases:

1. La desconstrucción. -El proceso de inferencia se genera fragmentando el discurso en sus microestructuras locales, las cuales se constituyen según van Dijk (1991) a partir de "la estructura de oraciones y las relaciones de conexión y coherencia entre ellas" (p.45), 
2. La reconstrucción discursiva. -En este punto se destaca la descripción y citas textuales de las microestructuras o subcategorías abordadas desde el método.

\section{Revisión de Literatura. Contexto y Antecedentes}

La organización, considerada como una agrupación deliberada de personas para el logro de algún propósito en específico se ha manifestado desde los albores de la humanidad, existen muchos tipos de organizaciones de carácter social, deportivo, políticos, etc.; pero en nuestro ámbito de trabajo se realizará un análisis organizacional, en tal virtud se considera como soporte en primera instancia las teorías organizacionales que sustenten la presente investigación. Como lo indica Neil Fligstein (1985) quien observa

...cada escuela de pensamiento ha tendido a concebir su teoría como una explicación causal total de los fenómenos organizacionales. Este hecho sugiere que una de las tareas centrales en teoría organizacional es la de reorientar el área de una manera tal que veamos las teorías competidoras como contribuyentes al entendimiento de los fenómenos organizacionales. (p. 377)

Es por esta razón que Pfeffer (1982) presenta un análisis de las distinciones de las diferentes escuelas del pensamiento él cual categoriza en teorías que versan sobre las acciones de individuos, coaliciones o sub-unidades organizacionales, fundamentados en premisas sobre las perspectivas que se adopten.

Un análisis orientado a un modelo de ecología de poblaciones y de dependencia de recursos; y en otro extremo modelos de acción organizacional basados en acciones intencionales y racionales, con énfasis en la dirección hacia objetivos bajo modelos de la contingencia racional y el modelo de costos de las transacciones, y por último la perspectiva sobre los actores organizacionales a través de sus valores creando un enfoque institucional de las organizaciones.

\section{El modelo de la ecología de poblaciones}

Este enfoque según Aldrich y Pfeffer (1976) propone que los factores ambientales seleccionan aquellas características organizacionales que mejor se adaptan al ambiente. El modelo de la ecología de las poblaciones también conocido como el enfoque de la selección natural se interesa primariamente en el cambio o la transformación organizacional, que no necesariamente sea un cambio a una mejor o más compleja organización sino la dirección del cambio sea hacia una mejor adaptación al ambiente.

El modelo de la ecología de las poblaciones según Aldrich y Pfeffer (1976) tiene tres estadios. El primero es aquel donde ocurre las variaciones en las formas organizacionales, las mismas que pueden o no ser planificadas, una vez que las variaciones han tenido lugar ocurre la selección. El segundo estadio, aquí una analogía de la evolución orgánica en la cual las mutaciones tiene éxito o no, dando una selección natural de aquellas que más se adecuen al ambiente.

El último estadio es el de la retención, es decir las formas organizacionales que son seleccionadas se preservan, duplican o se reproducen. Un ejemplo de esto serían las escuelas de negocios donde luego de plasmar las experiencias de organizaciones exitosas, estas sirven para la enseñanza y capacitación para nuevos gerentes

A este enfoque se han presentado ciertas observaciones, es así que: Freeman y Hannan (1989) son de la opinión de que el enfoque de la ecología se ajusta bien a la concepción de las organizaciones como sistemas complejos de limitada flexibilidad, pero Young argumenta que el enfoque puede ser apropiado solo para un rango restringido de fenómenos organizacionales.

El modelo de ecología de poblaciones tiende a prestar una imagen del ambiente en la que éste es desprovisto de actores humanos y es más bien una condición insensitiva, desinteresada en la que las organizaciones deben operar. Perrow (1979) ha observado también que el modelo elimina las variables en términos de poder, conflicto, desorden y clase social del análisis de los procesos sociales. 
Carroll (1984) ha ofrecido un útil conjunto de distinciones entre niveles de análisis. El asevera que el nivel organizacional puede ser utilizado en términos de proceso cíclicos de vida en las organizaciones. Al nivel de la ecología de las poblaciones se puede rastrear el crecimiento y declive de poblaciones enteras de organizaciones.

\section{El modelo de la dependencia de recursos}

La premisa básica del modelo de dependencia de recursos según Hasenfeld (1972) y Jacobs (1974) es, las decisiones son tomadas dentro de las organizaciones, las acciones son tomadas dentro del contexto político interno de las organizaciones, es decir en vez de ser receptoras pasivas de las fuerzas ambientales como se citaba en el anterior modelo, estas encaran a las condiciones ambientales en pro de sus planificaciones; si bien es cierto ninguna organización puede generar todos los recursos que necesita, tampoco puede realizar todas las actividades que se requiere para ser autosuficiente., $y$ sí se considera que existirán barreras que impedirán la movilización de estas organizaciones.

El modelo de dependencia por lo tanto se encarga de encarar un conjunto de posibles alternativas al tratar con su ambiente, "administrar sus ambientes lo mismo que sus organizaciones y la primera actividad puede ser tan importante e incluso más importante que la segunda" (Aldrich y Pfeffer, 1976, p.83). Lo que involucra que las decisiones son adoptadas de entre un conjunto de alternativas basada en la estrategia que la organización empleará intentando ser activa para influenciar en sus ambientes, ignorando los objetivos sino su actuar.

Aldrich y Pfeffer (1976), sobre la base de la obra de Child (1972) explica que existen tres formas en las cuales las opciones estratégicas operan en relación con el ambiente:

Primero, es la autonomía de quienes toman las decisiones, son los encargados de establecer que pautas sé seguirán para el posicionamiento de un segmento u otro, así como de permanecer en él o no.

Segundo, es aquella que en base a las decisiones tomadas se establecerán opciones estratégicas acerca del ambiente, es decir manipular al ambiente en sí, estas pueden crear demanda para sus productos, regularla legal o ilegalmente la competencia y confabular las tarifas y los precios si fuere necesario.

Tercero, es la fundamentada en la toma de decisiones estratégicas a través de la percepción y evaluación que tengan cada uno de los agentes decidores, es decir cada persona puede tener una lectura diferente sobre la realidad y por ende tomar decisiones acordes a su perspectiva.

Para finalizar es necesario recalcar que este modelo podrá verse enfocado en tácticas de como operar sus decisiones estratégicas y uno de los mecanismos más propicios para demostrar esto es la burocratización, entendiéndose aquella organización que se basa en los sistemas de archivo y documentación como precedentes para el presente organizacional, fundamentado en la especialización de roles, la estandarización, la descripción de puestos y por ultimo su estructura jerárquica que conduce a la forma más eficiente de la administración en virtud a la integración de los lazos interorganizacionales que actúa bajo la autoridad que es ejercida y no discute cada decisión.

\section{El modelo racional de la contingencia}

El modelo racional de la contingencia es un enfoque basado en los objetivos, que se enfocan en las razones por las cuales actúan y como lo hacen, alejándose de suposiciones acerca de la racionalidad o de un punto de vista simplista, al contrario, las organizaciones tienen múltiples y conflictivos objetivos.

La importancia de los objetivos según Simón (1964) argumenta que la idea de objetivos organizacionales es una reificación o un ejemplo de "tratar la organización como una entidad supraindividual con una conducta y existencia independiente de la conducta de sus miembros" (p.2).

El modelo racional de la contingencia como una forma de combinar los factores de las perspectivas

Fabara, Xavier 
de los diversos objetivos, conflictos y restricciones ambientales, basados en la teoría de las contingencias que de acuerdo a Scott (1981) "la manera óptima de organizar depende de la naturaleza del ambiente al cual debe relacionarse la organización” (p.114).

Este modelo según Katz y Kahn (1978) sugieren que la idea de una manera óptima de organizar para un ambiente particular ignora consideraciones políticas tales como demandas de negociación colectiva por un salario mínimo o por un acuerdo sindical. Es decir que la eficiencia muchas veces puede ser el resultado de medidas no necesariamente lo más justas.

El giro marxista ha adoptados esta postura en perspectiva diferente acerca del enfoque racional $\mathrm{y}$ por ende de la contingencia racional de las organizaciones. Pfeffer (1982)

El análisis marxista combina el determinismo ambiental con la opción estratégica racional. Después de todo Marx argumento que la evolución de los sistemas económicos era inevitable $\mathrm{y}$, en su análisis de las fuerzas sociales, concibió la presencia de ciertas tendencias históricas inexorables... Los análisis marxistas relevantes al entendimiento de las organizaciones han procedido desde la premisa de la acción estratégica racional, consciente adoptada por la clase de los capitalistas y por las organizaciones controladas por esta clase. (p.163)

\section{Goldman (1980)}

La posición marxista concibe un alto grado de consciencia e intencionalidad gerencial casi omnipotente no solo en las decisiones técnicas sino también en términos de programas ostensiblemente benignos tales como la acción benefactora de principios del siglo XX o de los experimentos de democratización de los 60 ' (p.12)

Con lo expuesto es útil considerar dos asuntos organizacionales importantes como lo describe Pfeffer: el primero es del control de los trabajadores y las relaciones, y el segundo se dirige a las relaciones interorganizacionales basada en la "racionalidad de clase" Useen (1979) existe cuando las elites buscan mejorar y proteger su posición a través de su participación activa en el gobierno de las organizaciones.

El modelo racional de la contingencia no necesariamente asume que la racionalidad tenga que ser alcanzada, así como sus objetivos pueden ser alcanzados o no y el ambiente donde se desenvuelve ser o no confrontado; en virtud que las organizaciones, que dan paso a la trasformación de propiedades, relaciones y acciones humanas; en propiedades, relaciones y acciones de cosas producidas por el hombre; objetos que se han vuelto independientes creando, como afirma Theodor Adorno que la sociedad y la conciencia han sido completamente cosificadas a través de este modelo las prácticas y las relaciones humanas llegan a ser vistos como objetos externos.

\section{El modelo de los costos de las transacciones}

El modelo de los costos de las transacciones surge del campo de la economía, Oliver Williamsom (1985) sugiere que este modelo trata de explicar la existencia y operación de las organizaciones particularmente las del sector privado. Teniendo como punto de partida el del intercambio o transacción de bienes y servicios, asumiendo que los individuos actúan en beneficio de sus intereses particulares y en un mercado libre.

\section{Scott (1987)}

En este modelo las variaciones en estructura organizacional fueron completamente irrelevantes. En contraste, la perspectiva de los costos de las transacciones asume que lo que es crítico no es la producción sino el intercambio de bienes y servicios, y enfatiza la importancia de las estructuras que gobiernan estos intercambios. (p.149)

\section{Granovetter (1985)}

El critica la perspectiva de los mercados y 
las jerarquías sobre la base de su concepción de que las transacciones económicas están en realidad inmersas en relaciones sociales. Desarrolla la posición de que en las sociedades modernas las transacciones económicas están ligadas a la confianza la cual, a su vez, está ligada a las relaciones sociales antes que a las relaciones económicas. (p.397)

Para concluir, este modelo se fundamenta en la ley de un libre mercado donde el objetivo primordial es la compra venta de productos y las estructuras se sujetarán a las exigencias de estas premisas, por lo tanto, puede ser empleado en combinación con cualquiera de los anteriores modelos en especial si se busca aportar al conocimiento sobre el origen de las organizaciones.

\section{El modelo institucional}

El modelo institucional emerge para contribuir al entendimiento de fenómenos organizacionales, el mismo que trata de explicar porque las organizaciones acogen determinadas formas, es por eso que DiMaggio y Powell (1983) arguyen que el "isomorfismo institucional" es hoy en día la razón dominante por la cual dichas organizaciones asumen las formas que tienen. Hay que tomar en cuenta también que la mayoría de organizaciones con este tipo de modelo son no lucrativas, y su análisis se basa en la suposición de que las organizaciones existen en campos formados por otras organizaciones que le son similares.

El concepto de campo organizacional nos referimos a aquellas organizaciones que, en el agregado, constituyen un área reconocida de la vida institucional: suplidores de claves, consumidores de recursos y productos, agencias regulatorias y otras organizaciones que producen servicios y productos similares. La virtud de esta unidad de análisis radica en que dirige nuestra atención no simplemente hacia las firmas competidoras o a redes interorganizacionales, sino hacia la totalidad de actores relevantes. (DiMaggio y Powell 1983, p.148)
Por lo que se entiende que las organizaciones cada vez son más homogéneas dentro de los campos, pero con sus respectivos isomorfismos que según DiMaggio y Pewell pueden explicarse por tres razones: la primera por las fuerzas coercitivas del ambiente así por ejemplo las regulaciones estatales, la cultura etc., la segunda son las incertidumbres que tiene que encarar las organizaciones. Y la tercera razón del isomorfismo es por las presiones normativas que se originan en la progresiva profesionalización de la fuerza laboral y en especial de los gerentes. Que a medida que se capacitan irán tomando decisiones homogéneas.

Es decir que "el diseño organizacional no como un proceso racional sino más bien como uno debido a presiones tanto externas como internas que, con el tiempo llevan a las organizaciones en un campo a parecerse unas a otras”. (Hall 1990, p.399).

Para concluir, este modelo los autores citan cuatro problemas que pueden tener serias consecuencias para la teoría institucional:

Primero. Un razonamiento tautológico potencial, es decir un razonamiento circular en el cual las variables son definidas cada una en términos de las demás haciendo difícil establecer las causas y los efectos

Segundo. La teoría no ha prestado casi atención alguna a la tarea de definir qué es lo que se encuentra institucionalizado y que no, ya que no todo lo que es llamado institucional esta institucionalizado.

Tercero. Es esencialmente ontológico; en razón que ciertamente se desarrollan mitos organizacionales individuales y colectivos acerca de los significados de esas realidades.

Finalmente es sobre la extensión, constituyéndose esta tendencia a la aplicación en una gran variedad de situaciones y organizaciones, DiMaggio y Powell (1983) fueron cuidadosos al hipotetizar que los efectos institucionales eran más probables en situaciones caracterizadas por una tecnología indeterminada y objetivos ambiguos. 


\section{Hallazgos}

A continuación, se presenta una sintetización de los modelos organizacionales analizados, conforme a la tabla $\mathrm{N}^{\mathrm{o}}$ 1, la cual explica su enfoque, el concepto, las características y observaciones en cada uno de los mencionados modelos. En base a los planteamientos de los siguientes autores: Pfeffer (1982), Aldrich y Pfeffer (1976), Freeman y Hannan (1989), Perrow (1979), Hasenfeld (1972), Jacobs (1974), Child (1972), Simón (1964), Scott (1981), Katz y Kahn (1978), Goldman (1980), Useen (1979), Williamsom (1985), Granovetter (1985), DiMaggio y Powell (1983), Hall 1990. 
Tabla 1

Articulación de los Modelos de las Tendencias de Estudios Organizacionales.

\begin{tabular}{|c|c|c|c|c|c|}
\hline MODELOS & $\begin{array}{l}\text { EL MODELO DE LA } \\
\text { ECOLOGÍA DE } \\
\text { POBLACIONES }\end{array}$ & $\begin{array}{l}\text { EL MODELO DE LA } \\
\text { DEPENDENCIA DE } \\
\text { RECURSOS }\end{array}$ & $\begin{array}{l}\text { EL MODELO } \\
\text { RACIONAL DE LA } \\
\text { CONTINGENCIA. }\end{array}$ & $\begin{array}{l}\text { EL MODELO DE LOS } \\
\text { COSTOS DE LAS } \\
\text { TRANSACCIONES }\end{array}$ & $\begin{array}{l}\text { EL MODELO } \\
\text { INSTITUCIONAL }\end{array}$ \\
\hline ENFOQUES & $\begin{array}{l}\text { enfoque de la selección } \\
\text { natural }\end{array}$ & $\begin{array}{l}\text { Enfoque de la } \\
\text { burocratización }\end{array}$ & basado en los objetivos & $\begin{array}{l}\text { enfoque del campo de la } \\
\text { economía }\end{array}$ & $\begin{array}{l}\text { enfoque del } \\
\text { isomorfismo } \\
\text { institucional }\end{array}$ \\
\hline CONCEPTO & $\begin{array}{l}\text { los factores ambientales } \\
\text { seleccionan aquellas } \\
\text { características } \\
\text { organizacionales que } \\
\text { mejor se adaptan al } \\
\text { ambiente }\end{array}$ & $\begin{array}{l}\text { las decisiones son } \\
\text { tomadas dentro de las } \\
\text { organizaciones, de } \\
\text { acuerdo al contexto } \\
\text { político interno de las } \\
\text { organizaciones }\end{array}$ & $\begin{array}{l}\text { explica las razones por } \\
\text { las cuales actúan y } \\
\text { como lo hacen las } \\
\text { organizaciones }\end{array}$ & $\begin{array}{l}\text { se fundamenta en el } \\
\text { intercambio o transacción } \\
\text { de bienes y servicios, }\end{array}$ & $\begin{array}{l}\text { explicar porque las } \\
\text { organizaciones } \\
\text { acogen determinadas } \\
\text { formas }\end{array}$ \\
\hline \multirow{3}{*}{ 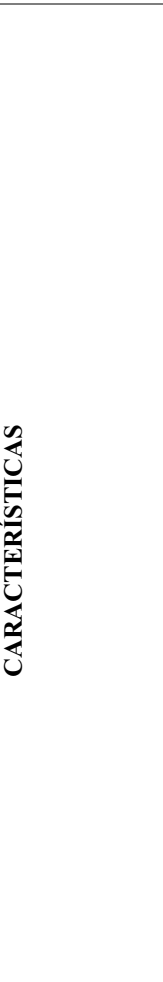 } & $\begin{array}{l}\text { variaciones en las } \\
\text { formas organizacionales }\end{array}$ & $\begin{array}{l}\text { autonomía de quienes } \\
\text { toman las decisiones }\end{array}$ & $\begin{array}{l}\text { la organización como } \\
\text { una entidad } \\
\text { supraindividual con una } \\
\text { conducta y existencia } \\
\text { independiente de la } \\
\text { conducta de sus } \\
\text { miembros }\end{array}$ & $\begin{array}{l}\text { explica la existencia y } \\
\text { operación de las } \\
\text { organizaciones } \\
\text { particularmente las del } \\
\text { sector privado }\end{array}$ & $\begin{array}{l}\text { las organizaciones } \\
\text { cada vez son más } \\
\text { homogéneas dentro } \\
\text { de los campos }\end{array}$ \\
\hline & $\begin{array}{l}\text { analogía de la evolución } \\
\text { orgánica }\end{array}$ & $\begin{array}{l}\text { las decisiones tomadas } \\
\text { se establecerán opciones } \\
\text { estratégicas acerca de } \\
\text { cómo actuar en el } \\
\text { ambiente }\end{array}$ & \multirow[t]{2}{*}{$\begin{array}{l}\text { La posición marxista } \\
\text { concibe un alto grado de } \\
\text { consciencia e } \\
\text { intencionalidad } \\
\text { gerencial casi } \\
\text { omnipotente no solo en } \\
\text { las decisiones técnicas } \\
\text { sino también en } \\
\text { términos de programas }\end{array}$} & $\begin{array}{l}\text { asume que lo que es } \\
\text { crítico no es la producción } \\
\text { sino el intercambio de } \\
\text { bienes y servicios, y } \\
\text { enfatiza la importancia de } \\
\text { las estructuras que } \\
\text { gobiernan estos } \\
\text { intercambios }\end{array}$ & $\begin{array}{l}\text { el diseño } \\
\text { organizacional no } \\
\text { como un proceso } \\
\text { racional, Sino como } \\
\text { un proceso adaptativo } \\
\text { de presiones externas } \\
\text { e internas que } \\
\text { conllevan a un campo } \\
\text { a parecerse unas a } \\
\text { otras }\end{array}$ \\
\hline & $\begin{array}{l}\text { organizaciones que son } \\
\text { seleccionadas se } \\
\text { preservan, duplican o se } \\
\text { reproducen }\end{array}$ & $\begin{array}{l}\text { la toma de decisiones } \\
\text { estratégicas a través de } \\
\text { la percepción y } \\
\text { evaluación que tengan } \\
\text { cada uno de los agentes } \\
\text { decidores }\end{array}$ & & $\begin{array}{l}\text { las sociedades modernas } \\
\text { las transacciones } \\
\text { económicas están ligadas a } \\
\text { la confianza la cual, a su } \\
\text { vez, está ligada a las } \\
\text { relaciones sociales antes } \\
\text { que a las relaciones } \\
\text { económicas }\end{array}$ & $\begin{array}{l}\text { Razonamiento } \\
\text { tautológico potencial; } \\
\text { La teoría no ha } \\
\text { definido ra } \\
\text { institucionalidad; Es } \\
\text { esencialmente } \\
\text { ontológico y objetivos } \\
\text { ambiguos. }\end{array}$ \\
\hline
\end{tabular}

\begin{tabular}{|c|c|c|c|c|c|}
\hline 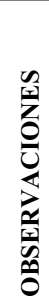 & $\begin{array}{l}\text { condición insensitiva, } \\
\text { desinteresada en la que } \\
\text { las organizaciones } \\
\text { deben operar }\end{array}$ & $\begin{array}{l}\text { fundamentado en la } \\
\text { especialización de roles, } \\
\text { la estandarización, la } \\
\text { descripción de puestos y } \\
\text { por ultimo su estructura } \\
\text { jerárquica }\end{array}$ & $\begin{array}{l}\text { las elites buscan mejorar } \\
\text { y proteger su posición a } \\
\text { través de su } \\
\text { participación activa en } \\
\text { el gobierno de las } \\
\text { organizaciones }\end{array}$ & $\begin{array}{l}\text { Los individuos actúan en } \\
\text { beneficio de sus intereses } \\
\text { particulares y en un } \\
\text { mercado libre. }\end{array}$ & $\begin{array}{l}\text { fuerzas coercitivas del } \\
\text { ambiente, las } \\
\text { incertidumbres que } \\
\text { tiene que encarar las } \\
\text { organizaciones y la } \\
\text { progresiva } \\
\text { profesionalización de } \\
\text { la fuerza laboral }\end{array}$ \\
\hline
\end{tabular}

Fuente: Elaboración propia a partir de la revisión de la literatura. 
Es necesario agregar que el estudio de los diferentes modelos organizacionales gira alrededor del porque las organizaciones actúan de una manera determinada, éste análisis podría concebirse como un problema de investigación, al tratar de comprender la realidad organizacional en sus múltiples facetas.

Las mismas que siempre van a estar íntimamente ligadas a los modelos, proceso y técnicas administrativas, por eso es importante aportar a este análisis, y que sirva como punto de partida para futuras investigaciones el gran aporte que realiza Ramírez
Martínez, Vargas Larios y De la Rosa (2011). Donde se establece los conocimientos generados de su investigación en función de la combinación de estos dos elementos del conocimiento: La administración y los estudios organizacionales, que lo resume en la tabla $\mathrm{N}^{\mathrm{o}} 2$.

De igual manera, el análisis cronológico que se establece en la figura $\mathrm{N}^{0} 1$ con respecto a la evolución de la teoría de la organización y los estudios organizacionales como un sustento teórico de la administración.

Tabla 2

Conocimientos generados por los Estudios Organizacionales y la Administración.

\begin{tabular}{ll}
\hline $\begin{array}{l}\text { Líneas de investigación y/o escuelas de los Estudios } \\
\text { Organizacionales }\end{array}$ & $\begin{array}{l}\text { Modelos, procesos y técnicas administrativas } \\
\text { comprendidas en la Administración }\end{array}$ \\
\hline Cultura en y alrededor de las organizaciones P & $\begin{array}{l}\text { roceso Administrativo } \\
\text { laneación estratégica }\end{array}$ \\
$\begin{array}{l}\text { Identidad organizacional P } \\
\text { Poder en y alrededor de las organizaciones D } \\
\text { Análisis Estratégico }\end{array}$ & $\begin{array}{l}\text { Desarrollo organizacional } \\
\text { Ambigüedad e incertidumbre en las organizaciones C }\end{array}$ \\
Nuevo Institucionalismo (económico, sociológico y político) & Administración de funciones (recursos humanos, \\
& mercadotecnia, finanzas, producción) \\
Ecología Organizacional A & dministración por objetivos \\
Aprendizaje y conocimiento en las organizaciones A & dministración estratégica y administración del \\
Psicoanálisis en las organizaciones T & conocimiento \\
\hline Líneas de investigación y/o escuelas de los Estudios & ecnologías de la información \\
Organizacionales & Modelos, procesos y técnicas administrativas \\
\hline Antropología de las organizaciones A & comprendidas en la Administración \\
Análisis postmoderno de las organizaciones C & dministración total de la calidad \\
Organizaciones Postmodernas & ultura corporativa \\
Transferencia de modelos organizacionales B & Lean Production y reingeniería \\
Cambio organizacional E & enchmarking \\
\hline
\end{tabular}

Fuente: Ramírez, Martínez, Vargas (2007). 
Tabla 3

Conocimientos generados por los Estudios Organizacionales y la Administración.

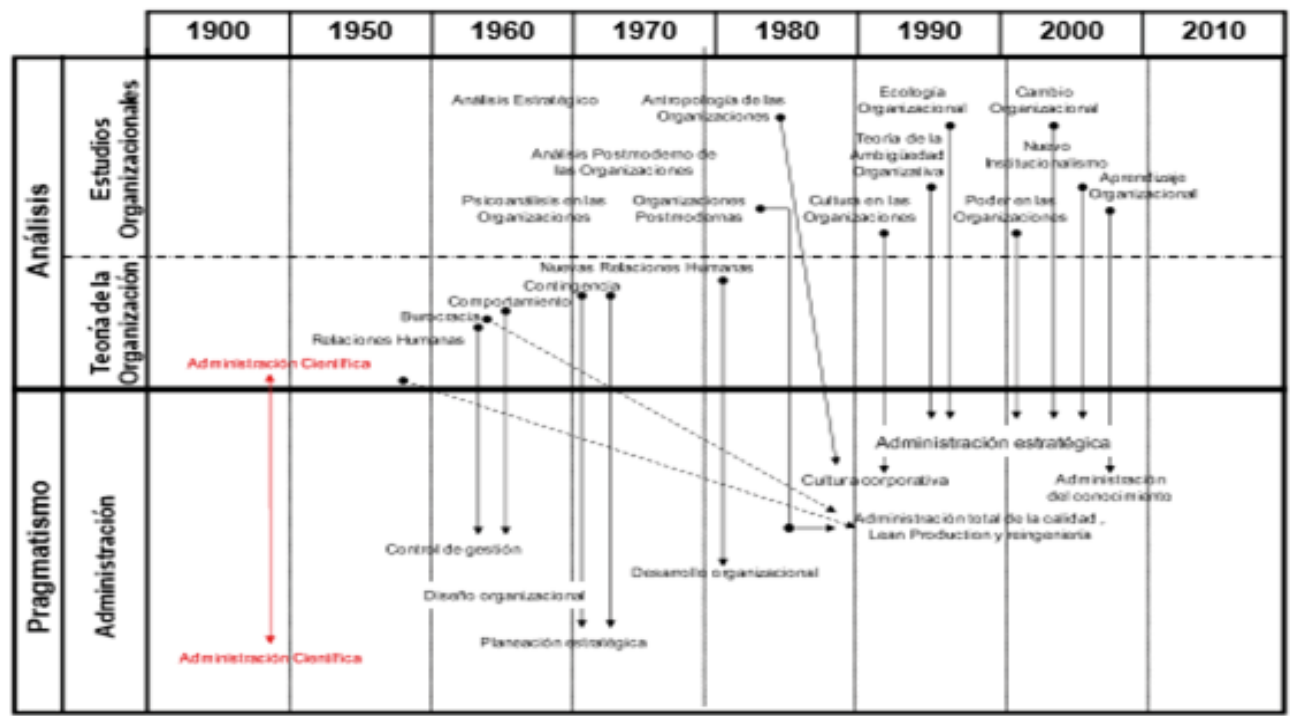

Fuente: Ramírez, Martínez, Vargas con base en De la Rosa, Lozano y López (2007).

De este análisis se puede colegir que como factor primordial de entre otros muchos factores, serán las tendencias de las estructuras organizacionales que cada vez serán más acordes al medio y a los objetivos que éstas persigan, siendo las tecnologías un factor muy importante para esa transformación de sistemas más abiertos y estructuras más planas y flexibles adaptables a su ambiente tanto como el micro entorno como el macro entrono que cada una de estas organizaciones se desenvuelva.

\section{Conclusiones}

Una organización, se considera a una agrupación deliberada de personas, para el logro de algún propósito en específico; las cuales se ha manifestado desde los albores de la humanidad, existen muchos tipos de organizaciones de carácter social, deportivo, políticos, etc.

El análisis organizacional, se enfoca a las interrelaciones que existen en la administración de una organización sea esta de carácter con fines de lucro o sin fines de lucro, las cuales consideran como soporte a las diferentes teorías organizacionales que sustenten su desarrollo.

Las revisiones teóricas indican un cambio de las tendencias del desarrollo organizacional, si consideramos a las organizaciones como un sistema vivo, este seguirá evolucionando tanto por factores o fuerzas internas, como externas; cada uno de los modelos describe varios puntos de vista de ese, su accionar; y el objetivo de este trabajo no es determinar cuál tenga o no mayor probabilidad de éxito o fracaso, cada una de ellas tiene su ámbito y su desempeño acorde a las realidades que se le han presentado.

Lo que sí se puede apreciar es que la mayoría de organizaciones se encuentran un mismo ambiente general, influencias especialmente por los avances tecnológicas y los niveles competitivos del mercado, que los conduce a ciertas tendencias, las mismas que indican que a futuro probablemente se consoliden estructuras de sistemas abiertos y más flexibles, más planas, virtuales y en red, todo pretendiendo llegar a ser organizaciones más eficientes, espontaneas, desestandarizadas, e innovadoras.

\section{Referencias bibliográficas}

Armenteros Acosta, M. D. C., Guerrero Ramos, L., Noyola del Rio, F. G., \& Molina Morejón, V. M. (2012). Cultura Organizacional Y Organización Que Aprende Un Análisis Desde La Perspectiva De La InnovaciónOrganizational (Culture and the Learning Organization: An Analysis from Innovation Perspective). Revista internacional

Fabara, Xavier 
administración \& finanzas, 5(1), 33-51.

Bustamante Bustamante, C. A. (2019). Prácticas de gestión humana en una PYME del sector de servicios (Doctoral dissertation, Especializacion en psicologia organizacional).

Cabañas, M. A. (Ed.). (2019). Tendencias organizacionales y contables contemporáneas. U. Externado de Colombia.

Escobar Trujillo, M. A., Jaramillo Pérez, Y. M., \& Mejía Muñoz, J. (2019). Juntos somos escuela. Aportes al mejoramiento de las relaciones laborales y académicas de la comunidad universitaria de la Escuela de Microbiología.

Espinoza, L. I. V. (1997). Consideraciones Epistemológicas de la Teoría Sociológica y de la Organizacional. Revista de Ciencias Sociales (Cl), (7), 62-71.

Estrada, F. (2008). Economía y racionalidad de las organizaciones. Los aportes de Herbert A. Simon. Revista de Estudios Sociales, (31), 84103.

Gallego, F. E. (2007). Herbert A. Simon y la economía organizacional. Cuadernos de Economía, 26(46).

Hall, R. H. (1990). Desarrollos recientes en teoría organizacional: una revisión. Ciencia y sociedad.

Madero-Gómez, S. M., \& Olivas-Luján, M. R. (2016). Análisis de los factores del comportamiento organizacional en jóvenes que están iniciando su carrera laboral. Estudios Gerenciales, 32(138), 51-59.

Pérez, A. M. S. (2016). Aproximación teórica a la evolución, teorías, enfoques y características que han sustentado el desarrollo de las organizaciones. Revista Cubana de Salud Pública, 42(4), 4.

Pérez, A. M. S. (2016). Desarrollo organizacional. Una mirada desde el ámbito académico. Educación Médica, 17(1), 3-8.

Rincón, D., \& Romero, M. G. (2002). Tendencias organizacionales de las empresas. Revista Venezolana de Gerencia, 7(19), 355-374.

Román-Calderón, J. P., Krikorian, A., Ruiz, C. F., \& Gaviria, A. B. (2016). Apoyo organizacional y empoderamiento como antecedentes de comportamientos empoderados y participación de los empleados. Estudios Gerenciales, 32(139), 154-161.

Salas-Arbeláez, L., Solarte, M. G., \& Vargas, G. M. (2017). Efecto de la cultura organizacional en el rendimiento de las pymes de Cali. Suma de Negocios, 8(18), 88-95.

Segredo Pérez, A. M. (2016). Aproximación teórica a la evolución, teorías, enfoques y características que han sustentado el desarrollo de las organizaciones. Revista Cubana de Salud Pública, 42, 585-595.

Terán Rosero, A. C. (2011). El capitalismo organizacional: una mirada a la calidad de vida laboral en la docencia universitaria. Cuadernos de Administración (Universidad del Valle), 27(46), 9-21.

Vargas, M. D. L. E. G., Sampieri, R. H., Álvarez, B. E. V., \& Vargas, M. H. C. (2012). Diagnóstico de la cultura organizacional en universidades tecnológicas bajo el Modelo de Valores en Competencia. Estudios, 2, 9-29.

Zamboni, L., \& Gorgone, H. R. (2003). Propuestas innovadoras en la gestión académica. 